\title{
NUMERICAL SIMULATION OF BLOOD FLOW IN LARGE VESSELS DURING THROMBUS FORMATION
}

Gábor Závodszky, György Paál

Budapest University of Technology and Economics, Department of Hydrodynamic Systems zavodszky@bds.bme.bu

\begin{abstract}
The thrombus formation in vessels is a very complex cascade process that is, amongst many other factors, dependent on the local blood flow characteristics. With the progressing formation, the geometry, and with it the flow pattern changes continuously. In addition to this, the adhesion, activation, and aggregation of the thrombocytes are heavily influenced by certain properties of the flow, most importantly by the emerging local shear forces. In the current work, the formation of a thrombus has been simulated using a simplified model of blood coagulation inside an artificial vessel section. While the employed blood coagulation model has heavily reduced the number of degrees of freedom compared to the real in-vivo bio-chemical processes, it is still capable of successfully reproducing several vital properties of a thrombus qualitatively.
\end{abstract}

\section{Introduction}

The hemostasis is a critical mechanism in the self-defense of our body. This is a natural response of the cardiovascular system that prevents undesirable blood loss in case of vascular injury. The blood coagulation is an important step during the hemostasis. It is a very complex cascade process driven by several factors that can both promote and inhibit it. The main steps include the aggregation and the binding of the platelets that are dependent on the local flow properties. Simulating this phenomenon is a challenging task as one has to make several appropriately chosen approximations. Anand et al. reviewed ${ }^{1}$ some of the interacting mechanisms regarding clot formation in flowing blood, and even though they deal with a narrower collection of

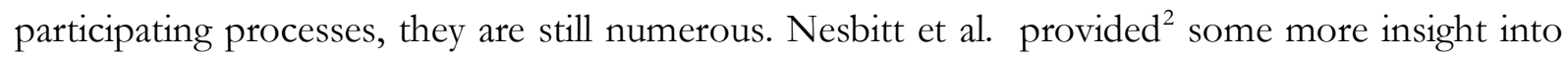
the thrombus formation by emphasizing the strong coupling with the hemodynamic properties. According to their experimental work, platelet aggregation is influenced by emerging shear forces as platelet adhesion favors low-shear zones. Several other authors recorded the ongoing process of thrombus formation: Celi et al. recorded ${ }^{3}$ the thrombus formation in the microcirculation of a living mouse using widefield video-microscopy. This process has also been recorded using several reagents to highlight the concentration of the main components of the formation. ${ }^{4-5}$

There are several works aiming at simulating thrombus formation numerically, but most of them cover only portions of the whole process. ${ }^{6-10}$ They try to solve the complex problem by approaching it either from the biochemical side and compute the reactions and the densities of the reagents, but fail to couple these equations with the changing flow field, or from the kinetic side, which usually lacks the appropriate handling of platelet concentrations. The platelet concentration profile inside vessels is an important factor, which is far from being constant in either venous or arterial vessels. ${ }^{11} \mathrm{~A}$ lot of numerical models have been developed in the past decade in order to calculate the platelet concentration profile. ${ }^{12-14}$ Their main findings are that the 
margination effect of platelets is mainly caused by the finite size effect of both the smaller platelets and the significantly larger red blood cells (RBC). The rolling motion of the larger RBCs pushes the platelets to the sides of the flow channel. This drift force that acts upon platelets in blood flow seems to be proportional with the average local flow velocity. To prove this interaction between platelets and RBCs, Mountrakis et al. simulated15 particle collisions in blood flow using the immersed boundary method.

\section{Methods}

During the simulations blood coagulation made the geometry change in time. For this reason, in our simulations a two-dimensional lattice Boltzmann based fluid solver was used on a $\mathrm{D}_{2} \mathrm{Q}_{9}$ lattice. This method has a clear advantage in handling complex and changing geometries over the more conventional finite-volume solvers. The theoretical bases of this method were laid down by Bhatnagar, Gross and Krook (BGK) ${ }^{16}$, and were later developed to the level of general usability for fluid flows by Quian et $\mathrm{al}^{17}$. We employed the so-called multiple-relaxation-time lattice Boltzmann model $^{18}$, which provides increased numerical stability compared to the widely used BGK model.

The platelets immersed in the blood flow were simulated as a passive scalar concentration field coupled with the velocity field of the flow capable of advection and diffusion. The passive attribute means that the platelet distribution has no influence on the blood flow. This approximation is supported by the fact that the usual volumetric ratio of the platelets in blood is under $1 \%$. The advection-diffusion of these platelets was also handled by the LBM method. When the velocity is prescribed by an already given velocity field (like in this case by the blood flow), the equilibrium function (from the original function ${ }^{17}$ ) of the scalar field reduces to the following:

$$
f_{i}^{e q}=\omega \rho\left(1+3 \overrightarrow{e_{l}} \cdot \vec{u}\right)(1)
$$

where $\omega$ is the relaxation frequency, $\overrightarrow{e_{l}}$ is the $i$-th discretized velocity direction, $\vec{u}$ is the local velocity of the blood flow, and $\rho$ is the platelet concentration that can be computed analogously to fluid density:

$$
\rho=\sum_{i=1}^{9} f_{i}
$$

where $f_{i}$ is the particle distribution along the $i$-th discretized velocity on the lattice. The diffusion coefficient $D$ then analogous to the numerical viscosity:

$$
D=\frac{1}{3}\left(\tau-\frac{1}{2}\right)
$$

where $\tau=\frac{1}{\omega}$ is the relaxation time for the equilibrium function. The rolling motion of the RBCs is mainly caused by the emerging shear forces in the fluid. To simulate the marginating effect, we prescribe a virtual force $\overrightarrow{F_{M}}$ that acts upon the density of the platelets. This force actually accounts for the finite size effects of the platelets and RBCs. The drift force acting on real platelets always drives platelets out from the main flow, to the sides of the vessel. It is a required behavior, as this causes increased platelet concentration in the vicinity of vessel walls where any 
injury can possibly occur. This $\overrightarrow{F_{M}}$ force points to the direction of the local largest shear stress decrease (in other words, to the direction of the smallest, most negative shear stress gradient). Its magnitude is proportional to the magnitude of the shear stress emerging in this specified direction. The proportionality ratio will require a proper parameter study later, although in the current work, it takes the value of one. Using this drift force, two simple channel flows were simulated with Reynolds numbers that are typical in these smaller vessels. The vessel wall was taken as a smooth, no-slip, rigid wall. The results of the simulations were compared to the experimental results of Woldhuis et al. ${ }^{17}$

The first simple channel flow simulation used constant velocity inlet and constant pressure outlet boundaries. The Reynolds number was set to one that is a typical value in venules. The result of the simulation is summarized below in Figure 1.

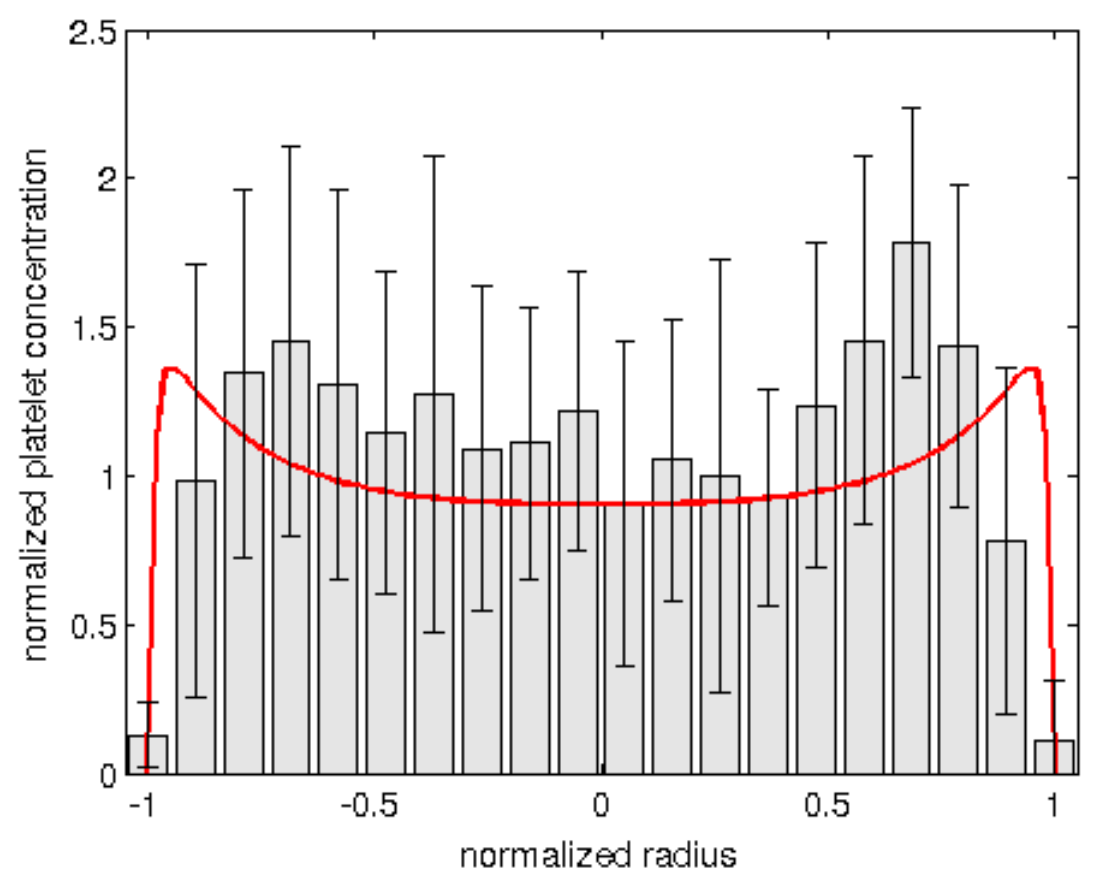

Figure 1. Normalized platelet concentration profile in a venule. The continuous red curve shows the results of the stationary simulation plotted over the experimental results of Woldhuis et al. ${ }^{19}(\mathrm{Re}=1)$

The platelet concentration shows a good qualitative agreement with the experimental results. This numerical model had to be tested with pulsatile flow as well, in order to reinforce the statement of its validity. For this reason, the other simulation used a time-varying inlet velocity boundary condition. Figure 2. shows the inlet flow rate profile that essentially mimics the behavior of a real cardiac pressure wave. 


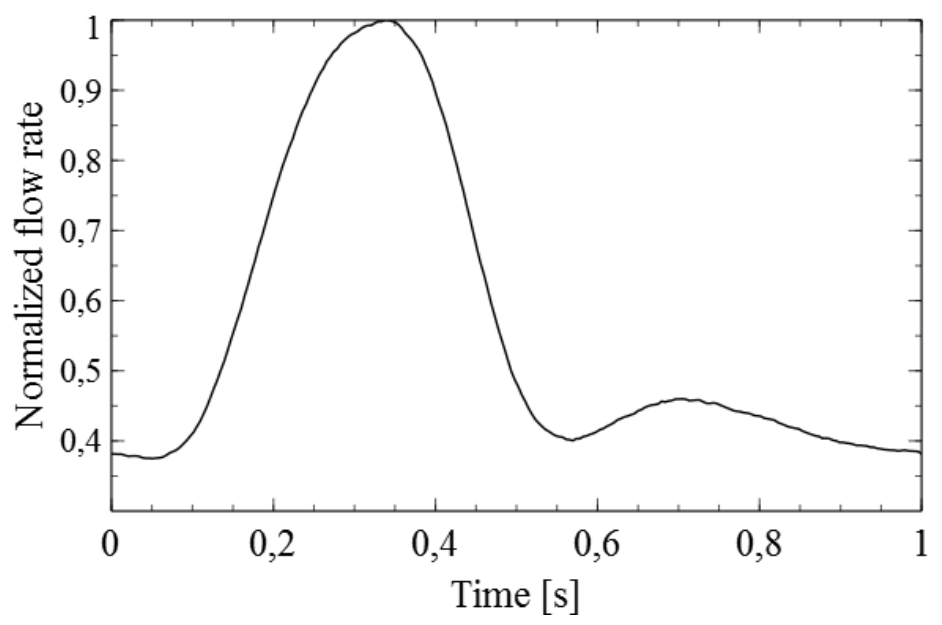

Figure 2. Normalized artificial flow rate curve that mimics the behavior of a real flow rate curve during a cardiac cycle.

The Reynolds number defined with the maximum of the possible inlet velocity was set to ten, which is a usual value for arterioles. The results of the simulation were also compared to the experimental results of the same authors in Figure 3. Again, good qualitative agreement has been found. The biggest deviation between the simulation and experimental results can be found near the vessel walls, but it is still within the error bars.

The coagulation of blood is modeled with changing the type of a numerical element from fluid cell to wall cell. For the thrombus formation in our simulation, coagulation is only possible at sites next to vessel walls or next to rigid blood cells that have already come to stasis. All the fluid LBM cells next to a wall cell have been tracked, as these cells have the ability to turn into a wall cell themselves. When this happens, their fluid cell neighbors are added to the list of near-wall cells. Though platelet activation in reality is a complex cascade process, we only simulate one reagent, the adenosine diphosphate (ADP) concentration, that is in our simulation not coupled with the blood flow. This means that the ADP concentration is not influenced by the local flow properties directly. Coagulation at a numeric lattice site depends on the relation of three variables:

$$
P_{\text {coag }}=\frac{\rho_{\text {platelet }} \cdot \rho_{A D P}}{\tau_{M A X}}
$$

where the $P_{\text {coag }}$ is a probability in the sense, that its value decides whether a fluid cell should come to stasis or not, based on the local platelet concentration $\rho_{\text {platelet }}$, the local ADP concentration $\rho_{A D P}$, and the local maximum shear component of the stress tensor denoted by $\tau_{M A X}$. The level of $P_{\text {coag }}$ that a numerical lattice has to reach for coagulation is an empirical parameter of the model for now. A later work should explore the deeper relation of these parameters. 


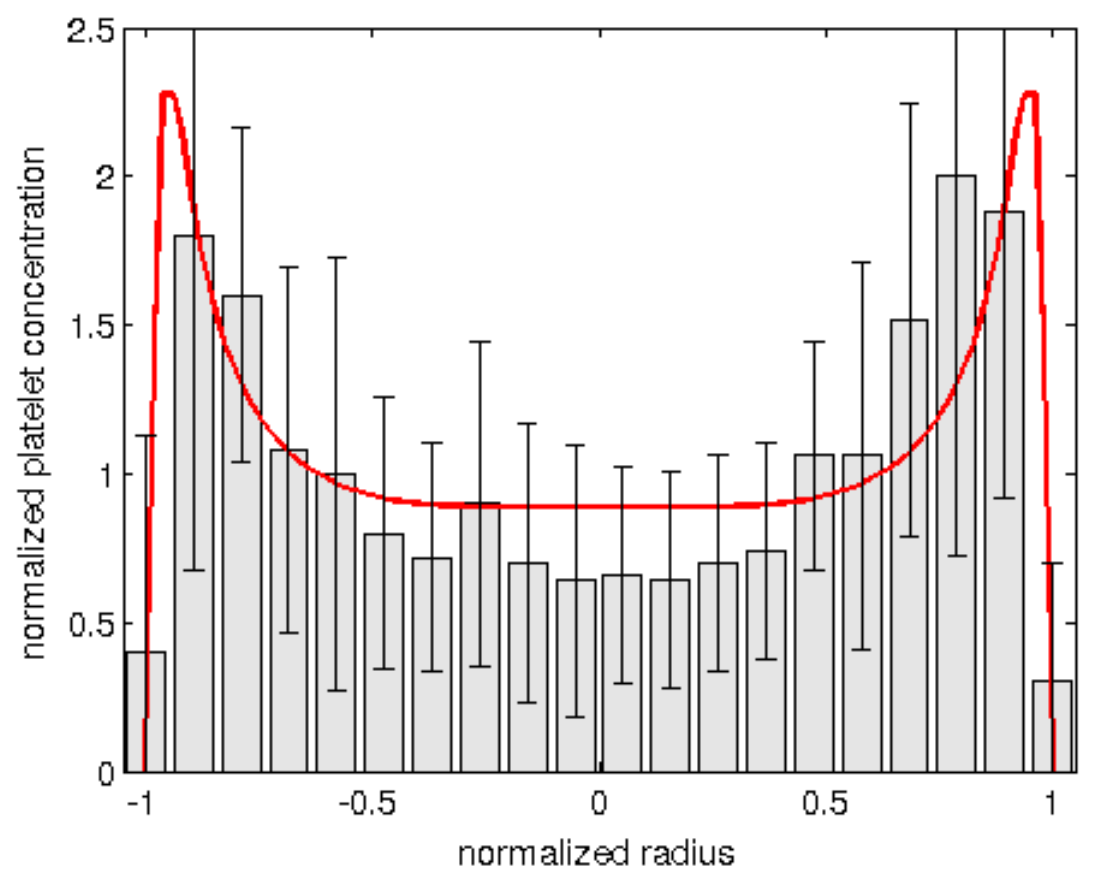

Figure 3. Time-averaged normalized platelet concentration profile in an arteriole. The continuous red curve shows the results of a transient simulation plotted over the experimental results of Woldhuis et al. ${ }^{19}$ $(\operatorname{Re}=10)$

There is one more component of the coagulation process. When a platelet is activated (or in our simulation when coagulation of a numerical lattice happens), it releases ADP that can initiate the activation of other platelets. In the simulations the ADP concentration decreases exponentially in space, so its release can be thought of as a nearly local ADP concentration increase. For coagulation, it is not enough to a single numerical lattice to have the appropriate values for one iteration, the parameters have to remain in the coagulation zone for a $t_{\text {window }}$ time. This time was chosen to be $t_{\text {window }}=20 \mathrm{~ms}$, because this is the usual timeframe for ADP to activate a platelet. This also means that a newly registered near-wall lattice cannot turn into a wall cell sooner than $20 \mathrm{~ms}$.

In Figure 4. a snapshot of a coagulation simulation is shown after six heartbeat cycles. The formation of the thrombus starts at an injury site. In in-vivo systems in case of vascular injury, a significant amount of ADP is released into the bloodstream from the injured vessel wall tissue. The injury site in this case was simulated by increasing the ADP concentration at a small amount of numerical lattices next to the vessel wall. 

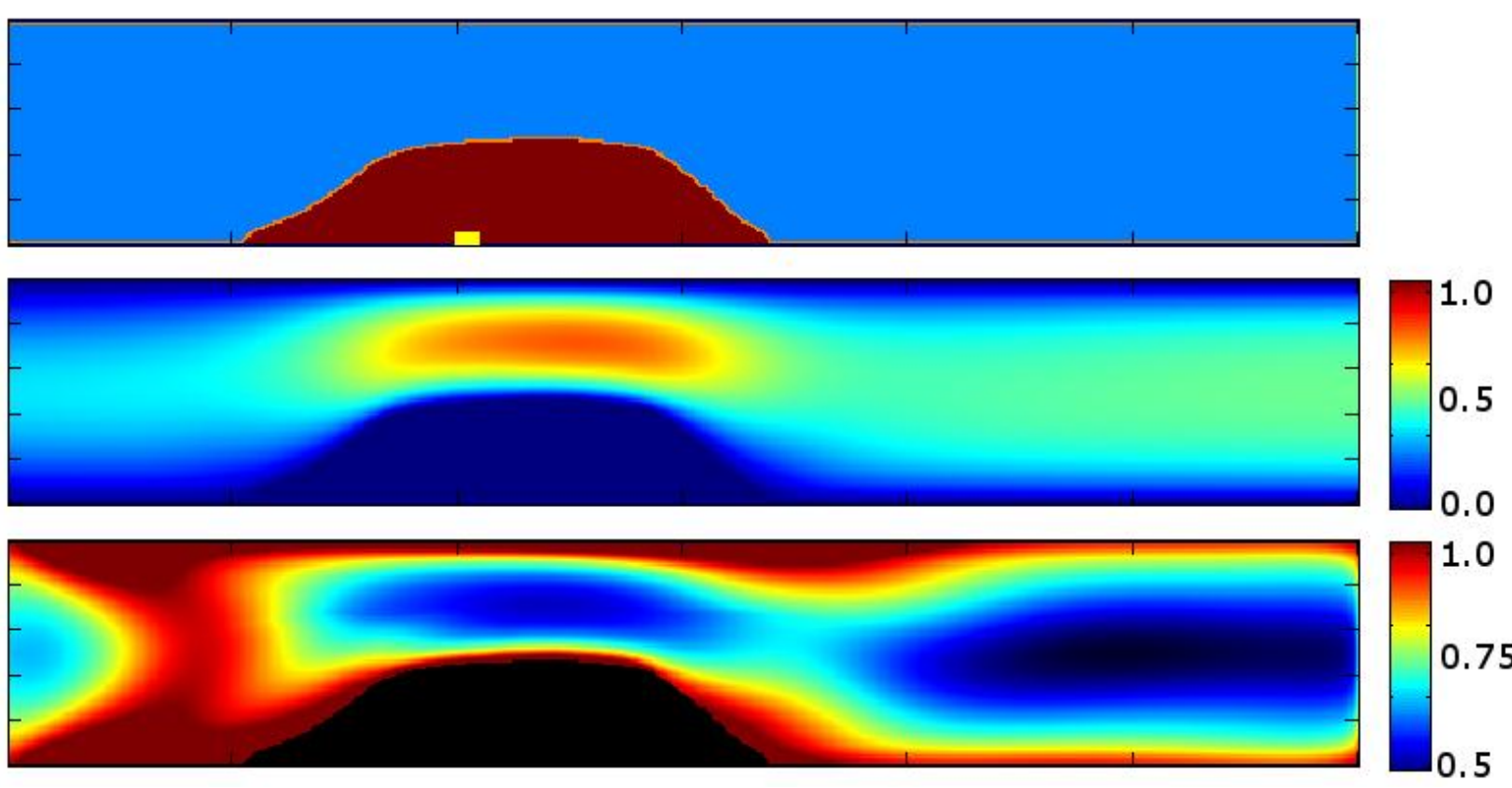

Figure 4. Typical result of a thrombus formation simulation at time instant of the end of a heartbeat cycle. The flow direction is from the left to the right. The upper image shows the emerged geometry of the thrombus, with the place of vessel injury (yellow box at the bases of the thrombus). The middle image shows the current normalized velocity magnitudes (Reynolds number equals 10). The lower image shows the normalized platelet concentration at the same instant.

There are a few qualitative features of the calculated thrombus that worth mentioning. Firstly, as the thrombus begins to block the flow channel, the flow speed increases and that leads to larger emerging shear stresses that inhibit the process of the coagulation. This causes the relatively flat top of the thrombus. It is also noticeable that the thrombus growth is asymmetric to the site of injury; its growth is slower on the side facing the incoming bloodstream. This is again the result of the different shear stresses.

Two resulting thrombus geometries recorded at different times were compared to the videomicroscopy results of Nesbitt et al. ${ }^{11}$ in Figure 5. The experimental results originate from an induced injury inside the arteriole of a living mouse. The exact form of the thrombus naturally depends on much more components that are taken into account in our simulation. Still, some qualitative features like effects of shear stresses can be studied with it.

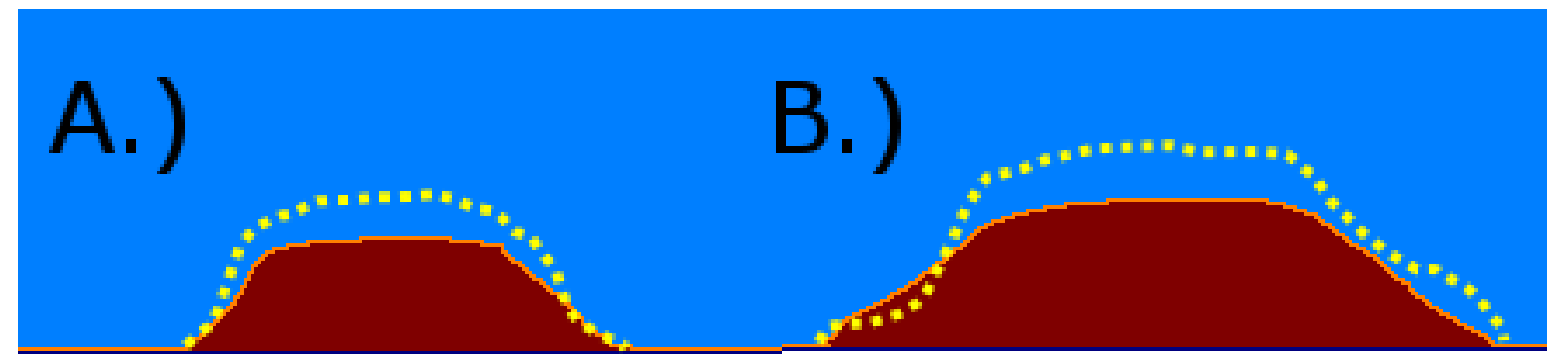

Figure 5. Geometry of the thrombus after A.) three and after B.) six heart beat cycles. The yellow dotted outline shows the geometry of the thrombus recorded with videomicroscopy by Nesbitt et al. ${ }^{2}$ 


\section{Conclusion}

Simulation of blood coagulation is a heavily studied topic, with wide possibilities for applications. In this work a numerical model was presented that is computationally effective due to the large number of approximations. And though this model evidently has some shortcomings, like that in videomicroscopy records the outer layer of the thrombus is often not solid, and can dissolve easily in the flow and subside again later. In our model, however, the thrombus is handled as solid material, unable to dissolve. Also the effects of the several components of the coagulation cascade that was not taken into account. The model still seems to succeed in reproducing the major characteristics of an injury induced thrombus by only using a few components and coupling them to the flow properties. It should also be mentioned that due to the strongly simplified model, it might be capable of simulating other sediments in blood flows, like the deposition of fat particles on clinical devices. This possibility will drive the research to explore the limits of validity of such a simple coupled numerical model further.

\section{REFERENCES}

1. Anand M, Rajagopal KR. A Model Incorporating some of the Mechanical and Biochemical Factors Underlying Clot Formation and Dissolution in Flowing Blood. Journal of Theoretical Medicine 5, 2003 p. 183-218.

2. Nesbitt W S, Westein E, Tovar-Lopez FJ, Tolouei E, Mitchell A, Fu J, Carberry J, Fouras A, Jackson S P. A shear gradient-dependent platelet aggregation mechanism drives thrombus formation. Nature Medicine 2009;15:665-73.

3. Celi A, Merrill-Skoloff G, Gross P, Falati S, Sim DS, Flaumenhaft R, Furie BC, Furie B. Thrombus formation: direct real-time observation and digital analysis of thrombus assembly in a living mouse by confocal and widefield intravital microscopy. Journal of thrombosis and haemostasis JTH 1, 2003 p. $60-68$.

4. Falati S, Gross P, Merrill-Skoloff G, Furie B C, Furie B. Real-time in vivo imaging of platelets, tissue factor and fibrin during arterial thrombus formation in the mouse. Nature Medicine 8, 2002 p. 117581.

5. Furie B, Furie B C. In vivo thrombus formation. Journal of thrombosis and haemostasis JTH 5 2007;(1):12-7.

6. Alenitsyn AG, Kondratyev AS, Mikhailova I, Siddique I. Mathematical Modeling of Thrombus Growth in Microvessels. Journal of Prime Research in Mathematics 2008;4:195-205.

7. Harrison S E, Smith S M, Bernsdorf J, Hose D R, Lawford P V. Application and validation of the lattice Boltzmann method for modelling flow-related clotting. Journal of Biomechanics 40, $2007 \mathrm{p}$. 3023-8.

8. Wang W, King MR. Multiscale Modeling of Platelet Adhesion and Thrombus Growth. Annals of Biomedical Engineering, 2012 November 2012;40(11):2345-54.

9. Tamagawa M, Kaneda H, Hiramoto M, Nagahama S. Simulation of thrombus formation in shear flows using Lattice Boltzmann Method. Artificial Organs 33, 2009 p. 604-10.

10. Wang W, King MR. Multiscale Modeling of Platelet Adhesion and Thrombus Growth. Annals of Biomedical Engineering 2012.

11.Zhao H, Shaqfeh ESG. Numerical simulation of the margination of platelets in the microvasculature. Center of turbulence research, Stanford, Annual Research Briefs, 2010. 
12. Jordan A, David T, Homer-Vanniasinkam S, Graham A, Walker P. The effects of margination and red cell augmented platelet diffusivity on platelet adhesion in complex flow. Biorheology 41, 2004. p. 64153.

13. Tokarev AA, Butylin AA, Ermakova EA, Shnol EE, Panasenko GP, Ataullakhanov FI. Finite platelet size could be responsible for platelet margination effect. Biophysical Journal 101, 2011. p. 1835-43.

14. Zhao R, Kameneva MV, Antaki JF. Investigation of platelet margination phenomena at elevated shear stress. Biorheology 44, 2007. p. 161-77.

15. Mountrakis L, Lorenz E, Hoekstra AG. Where do the platelets go? A simulation study of fully resolved blood flow through aneurysmal vessels. Interface Focus 3: 20120089, Royal Society Publishing, 2012.

16. Bhatnagar PL, Gross EP, Krook M. A Model for Collision Processes in Gases. I. Small Amplitude Processes in Charged and Neutral One-Component Systems. Physical Review 94, 1954. p. 511-25.

17. Qian YH, D' Humières D, Lallemand P. Lattice BGK Models for Navier-Stokes Equation. Europhysics Letters 17, 1992. p. 479-84.

18. D’Humières D, Ginzburg I, Krafczyk M, Lallemand P, Luo L-S. Multiple-relaxation-time lattice Boltzmann models in three dimensions. hilosophical Transactions of the Royal Society - Series A: Mathematical, Physical and Engineering Sciences 360, 2002. p. 437-51.

19. Woldhuis B, Tangelder GJ, Slaaf DW, Reneman RS. Concentration profile of blood platelets differs in arterioles and venules. American Journal of Physiology . 1992. 262, H1217-H1223. 